\title{
Analysis of strain fields in silicon nanocrystals
}

Dündar E. Yılmaz, Ceyhun Bulutay, and Tahir Çağın

Citation: Appl. Phys. Lett. 94, 191914 (2009);

View online: https://doi.org/10.1063/1.3138163

View Table of Contents: http://aip.scitation.org/toc/apl/94/19

Published by the American Institute of Physics

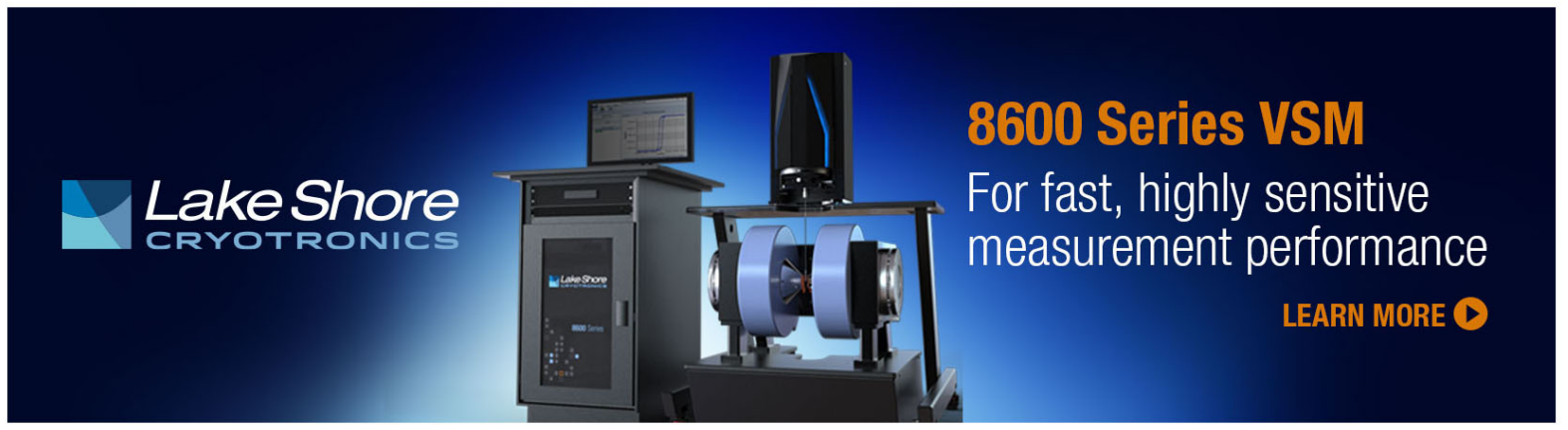




\title{
Analysis of strain fields in silicon nanocrystals
}

\author{
Dündar E. Yılmaz,, ${ }^{1, a)}$ Ceyhun Bulutay, ${ }^{1, b)}$ and Tahir Çağın ${ }^{2, c)}$ \\ ${ }^{1}$ Department of Physics, Bilkent University, Ankara 06800, Turkey \\ ${ }^{2}$ Artie McFerrin Department of Chemical Engineering, Texas A\&M University, Jack E. Brown \\ Engineering Building, 3122 TAMU, College Station, Texas 77843-3122, USA
}

(Received 29 December 2008; accepted 28 April 2009; published online 15 May 2009)

\begin{abstract}
Strain has a crucial effect on the optical and electronic properties of nanostructures. We calculate the atomistic strain distribution in silicon nanocrystals up to a diameter of $3.2 \mathrm{~nm}$ embedded in an amorphous silicon dioxide matrix. A seemingly conflicting picture arises when the strain field is expressed in terms of bond lengths versus volumetric strain. The strain profile in either case shows uniform behavior in the core, however, it becomes nonuniform within 2-3 $\AA$ distance to the nanocrystal surface: tensile for bond lengths whereas compressive for volumetric strain. We reconcile their coexistence by an atomistic strain analysis. (c) 2009 American Institute of Physics. [DOI: $10.1063 / 1.3138163$ ]
\end{abstract}

The low dimensional forms of silicon embedded in silica have strong potential as an optical material. ${ }^{1}$ Such heterogeneous structures inherently introduce the strain as a degree of freedom for optimizing their optoelectronic properties. It was realized earlier that strain can be utilized to improve the carrier mobility in bulk silicon based structures. ${ }^{2}$ This trend has been rapidly transcribed to lower dimensional structures, starting with two-dimensional silicon structures. ${ }^{3}$ Recently for silicon nanowires, there have been a number of attempts to tailor their optical properties through manipulating strain. ${ }^{4,5}$ Furthermore, recent studies have revealed that the strain can become the major factor restricting the crystallization of the nanolayers. ${ }^{6,7}$ It depends on several factors, most important of which are the lattice mismatch between the constituents, size of the nanocrystals (NCs), and the growth conditions, such as the details of the growth procedure. ${ }^{8}$ In summary, for improving the optical and electronic properties of $\mathrm{NCs}$, the strain engineering has become an effective tool to be exploited. ${ }^{9-11}$ A critical challenge in this regard is to analyze the strain state of the Si NCs embedded in silica.

The close relations between strain and optical or electronic properties in $\mathrm{Si} \mathrm{NCs}$ have very recently become the center of attention. ${ }^{10,12,13}$ There still remains much to be done in order to understand strain in nanostructures at the atomistic level. As pioneered by Tsu et al. ${ }^{14}$ Raman spectroscopy can be an effective experimental tool for determining the strain state of the Si NCs. Specifically, recent Raman studies reported that the Si NCs may be under a thermal residual strain and this can be reduced by overall annealing at high temperatures ${ }^{8}$ or by local laser annealing. ${ }^{15}$ Due to small density difference between $\mathrm{Si} \mathrm{NC}$ and the surrounding $a-\mathrm{SiO}_{2}$, a limited information can be gathered about its structure using transmission electron microscopy (TEM) or even high resolution TEM techniques. ${ }^{16}$ Especially, molecular dynamics (MD) simulations with realistic interaction potentials present an opportunity, by providing more detailed critical information then the best imaging techniques currently available and clarify the analysis of experimental results. Along this direction, previously ${ }^{17}$ we focused on $\mathrm{Si}-\mathrm{Si}$ bond length

\footnotetext{
${ }^{a)}$ Electronic mail: dundar@fen.bilkent.edu.tr.

${ }^{b)}$ Electronic mail: bulutay@fen.bilkent.edu.tr.

c)Electronic mail: cagin@chemail.tamu.edu.
}

distribution and reported that $\mathrm{Si}-\mathrm{Si}$ bond lengths are stretched up to 3\% just below the surface of Si NCs embedded in amorphous $\mathrm{SiO}_{2}$ which has also been very recently confirmed. ${ }^{18}$

In this letter, we analyze the volumetric and bond length strain distributions in Si NCs, in particular, demonstrate that both compressive volumetric strain and tensile bond length strain coexist within the same Si NC. We accomplish this by performing trajectory analysis on model samples (with $\sim 5000$ atoms) simulated via MD using a reliable and accurate as well as reactive force field. ${ }^{19}$ The simulation details are similar to our previous work, ${ }^{17}$ except the way we construct the Si NC in glass matrix. Instead of deleting all glass atoms within a predetermined radius, we remove the glass atoms after rigorously defining the surface of the nanocrystal through the Delaunay triangulation method. ${ }^{20}$ In this way, we have constructed NCs embedded in glass matrix with diameters ranging from 2.2 to $3.2 \mathrm{~nm}$ without introducing built-in strain to the system. In this diameter range we observe similar trends in strain, volumetric strain, and bond length distribution, etc., therefore, we present only the figures of the system for a typical $\mathrm{NC}$ with diameter of $2.6 \mathrm{~nm}$.

In the language of geometry, strain is defined through an affine transformation that maps the undeformed state to deformed state, which is called deformation gradient. Several methods to derive discrete form of deformation gradient from atomic positions are reported. ${ }^{21-23}$ In the method proposed by Pryor et al. ${ }^{23}$ the atomistic strain tensor is derived from local transformation matrix that transforms nearest neighbors of a certain atom from its undeformed state to the deformed one. From the MD simulations, using positions of $\mathrm{NC}$ atoms, we first extract each atoms' displacement vector from its undeformed site which is determined by positioning an ideal tetrahedron to the local environment. Using these displacement vectors, we construct deformation matrix and derive the atomistic strain tensor from this local deformation tensor. The first invariant of strain tensor corresponds to the hydrostatic strain. ${ }^{24}$ As an alternative measure to hydrostatic strain, we calculate volumetric strain by considering volume change of a tetrahedron from its undeformed counterpart. A third measure as we have used in our previous report, ${ }^{17}$ is the bond length strains. 


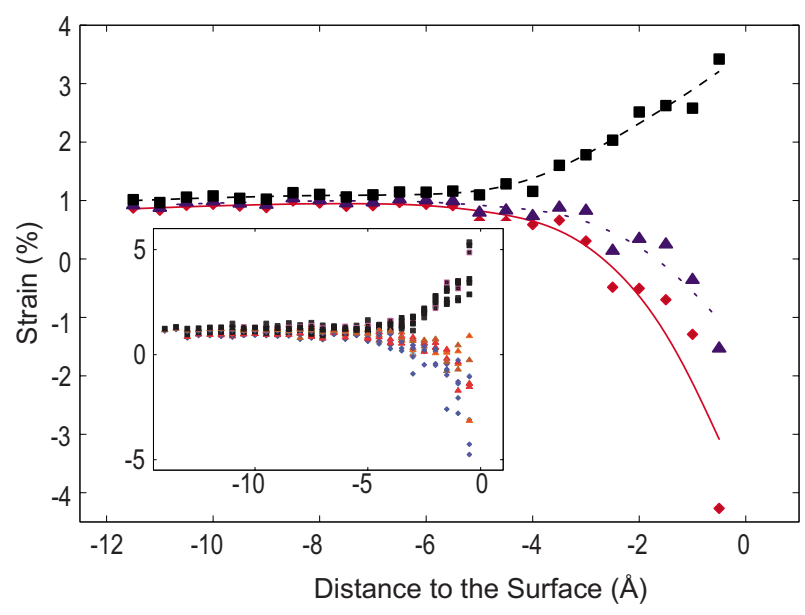

FIG. 1. (Color online) Variation of, Si-Si bond lengths (squares), hydrostatic strain (diamonds), and the volumetric strain (triangles) as a function of distance to NC surface (see text). Dashed, dotted, and solid lines are guides to the eye for the respective data set. The data for $2.6 \mathrm{~nm}$ diameter NC are used. Inset: Other NC diameters ranging from 2.2 to $3.2 \mathrm{~nm}$ are also shown.

To verify our results we have calculated strain distribution in NC region for all mentioned measures. We have plotted all three of them in Fig. 1. The results of volumetric strain are very close to hydrostatic strain which is the trace of strain tensor calculated with aforementioned technique. ${ }^{23}$ In these results, we observe a net compressive behavior of strain just under the surface and a uniform tensile strain of about $1 \%$ at the core of $\mathrm{NC}$. $\mathrm{Si}-\mathrm{Si}$ bonds are stretched by about $1 \%$ in the core region in agreement with the hydrostatic and volumetric strains, however, just under the surface, $\mathrm{Si}-\mathrm{Si}$ bonds are stretched up to $3 \%$ where hydrostatic and volumetric strain results indicate compressive strain state. The bond stretch in $\mathrm{Si}-\mathrm{Si}$ bonds due to oxidation has been shown earlier by us using MD simulations ${ }^{17}$ which was also confirmed by other approaches. ${ }^{18}$ Occurrence of compressive volumetric strain and stretched bond lengths in the same outer region may initially seem contradicting. However, stretching of bonds does not imply that the system is under tensile hydrostatic strain as well. Consider a tetrahedron formed by a Si atom and its four Si neighbors (A, B, C, D) as shown in upper inset of Fig. 2. In the ideal case, the solid angle $(\Omega)$ subtended by each triangular face of this tetrahedron should be equal to $180^{\circ}$. Under a uniform deformation, bond lengths will also be stretched, while the solid angles remain unchanged. However, under a nonuniform deformation, the change in three solid angles causes a decrease in the volume of the tetrahedron while increasing or preserving the bond lengths. Hence, a combination of stretched bond lengths with deformed solid angles may end up with an overall reduction in the volume of the tetrahedron. This explains the coexistence of compressive volumetric strain and stretched bond lengths at the region just below the surface of NCs.

To better visualize the nature of the deformation of the $\mathrm{Si}$ NCs, we consider the orientational variation of the solid angles of the tetrahedral planes. As illustrated in the lower inset of Fig. 2, the two important directions are the unit normal $\left(\hat{n}_{\mathrm{S}}\right)$ of the tetrahedron face subtending the solid angle under consideration, and the local outward surface normal $\left(\hat{n}_{\mathrm{NC}}\right)$ of the NC. It is clearly seen from Fig. 2 that solid angles subtended by tetrahedra faces oriented outward to the

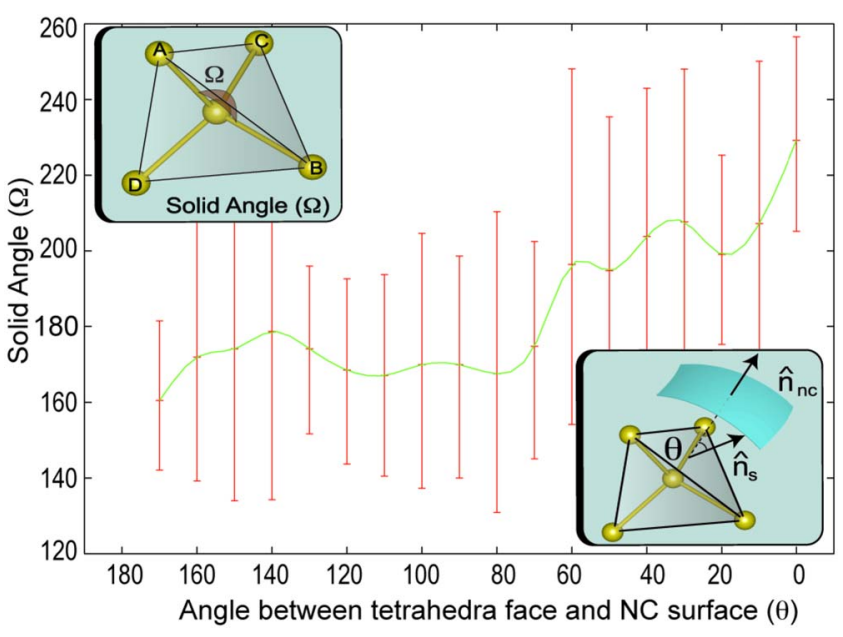

FIG. 2. (Color online) Dependence of solid angle subtended by tetrahedron face to the angle between tetrahedron face and NC surface. Illustration of solid angle subtended by tetrahedron face (top left inset) and the angle between tetrahedron face and NC surface (bottom right inset).

NC surface are increased up to $220^{\circ}$, whereas those facing inward to the $\mathrm{NC}$ core are decreased down to $160^{\circ}$. This dependence is a clear evidence of how oxidation affects strain distribution close to the interface.

To further quantify the atomistic strain in the highly critical region within $3 \AA$ distance to the interface, we classify the average bond length and hydrostatic strain behaviors into three categories. Figure 3 displays the percentage as well as the bonding details of each category. In top left, we illustrate most common type with a share of $53.0 \%$ which is responsible for the opposite behavior in Fig. 1 where average bond lengths of center Si atoms to its four nearest neighbors are stretched but net atomistic strain at this atom is compressive. In this case solid angles facing toward the oxide region are increased to $270^{\circ}$ due to oxygen bonds of Si neighbors.

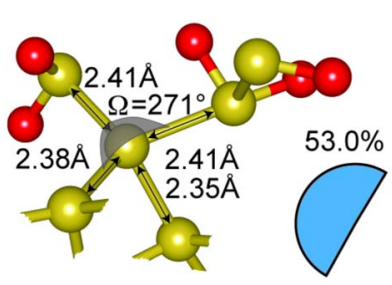

Hydrostatic Strain $=-2.7 \%$ Av. Bond Length $=5.2 \%$

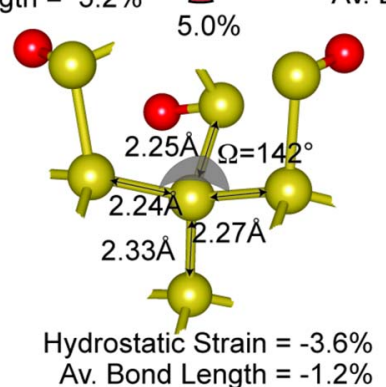

FIG. 3. (Color online) Illustrations of oxidation effects on strain in three categories with their percentage of occurrences: $\mathrm{Si}-\mathrm{Si}$ bonds are stretched and system is under compressive strain (upper left). Si-Si bonds are stretched and system is under tensile strain (upper right). Si-Si bonds are shortened and the Si atom at the center is under compressive strain (bottom). Large spheres (gold) and small spheres (red) represents Si and O atoms, respectively. 
Although these oxygen bonds stretched $\mathrm{Si}-\mathrm{Si}$ bonds to 2.41 $\AA$, net strain on center $\mathrm{Si}$ atom is $-2.7 \%$. In the top-right part of the Fig. 3 we illustrate second most often case with a percentage of $42.0 \%$, where average bond lengths and atomistic strain show similar behavior; bond lengths are stretched and net hydrostatic strain is tensile. In this case oxidation somewhat uniformly deforms the bonds so that solid angles are still around $180^{\circ}$ which is the value for the unstrained case. Finally, as shown at bottom of Fig. 3, a very small percentage of atoms $(5.0 \%)$ in the region beneath the surface have shortened bond lengths and compressive atomistic strain.

In summary, we study the strain state of Si NCs in silica matrix with diameters in 2-3.2 $\mathrm{nm}$. The structure is assumed to be free from any thermal built-in strains. The core region of the NC is observed to be under a uniform $1 \%$ tensile strain, where both bond length and volumetric strain measures are in agreement. However, toward the NC interface, while the $\mathrm{Si}-\mathrm{Si}$ bonds become more stretched, the hydrostatic strain changes in the compressive direction. In the interpretation of the indirect strain measurements, e.g., from spectroscopy, this dual character needs to be taken into consideration. We explain these two behaviors using the solid angle deformation of the tetrahedral-bonded $\mathrm{Si}$ atoms, and demonstrate that it is ultimately caused by the oxygen atoms at the interface. An equally important finding is that the overall strain profile within the Si NCs is quite nonuniform. As very recently emphasized, within the context of centrosymmetric materials, such as silicon, such strain gradients locally break the inversion symmetry and may lead to profound physical consequences. ${ }^{25}$

This work has been supported by the Turkish Scientific and Technical Council TÜBİTAK with the Project No. 106 T048 and by the European FP6 Project SEMINANO with the Contract No. NMP4 CT2004 505285. The visit of Tahir Çağın to Bilkent University was facilitated by the
TÜBİTAK BİDEB-2221 program. T.C. also acknowledges the support of NSF-IGERT.

${ }^{1}$ Nano Silicon, edited by V. Kumar (Elsevier, London, 2007).

${ }^{2}$ H. M. Manasevit, I. S. Gergis, and A. B. Jones, Appl. Phys. Lett. 41, 464 (1982).

${ }^{3}$ R. People, J. C. Bean, D. V. Lang, A. M. Sergent, H. L. Stormer, K. W. Wecht, R. T. Lynch, and K. Baldwin, Appl. Phys. Lett. 45, 1231 (1984).

${ }^{4}$ D. M. Lyons, K. M. Ryan, M. A. Morris, and J. D. Holmes, Nano Lett. 2, 811 (2002).

${ }^{5}$ K.-H. Hong, J. Kim, S.-H. Lee, and J. K. Shin, Nano Lett. 8, 1335 (2008).

${ }^{6}$ M. Zacharias and P. Streitenberger, Phys. Rev. B 62, 8391 (2000).

${ }^{7}$ S. Hernandez, A. Martinez, P. Parinello, Y. Lebour, B. Garrido, E. Jordana, and J. M. Fedeli, J. Appl. Phys. 104, 044304 (2008).

${ }^{8}$ M. Zacharias, J. Bläsing, P. Veit, L. Tsybeskov, K. Hirschman, and P. M. Fauchet, Appl. Phys. Lett. 74, 2614 (1999).

${ }^{9}$ A. Thean and J. P. Leburton, Appl. Phys. Lett. 79, 1030 (2001).

${ }^{10}$ X. H. Peng, S. Ganti, A. Alizadeh, P. Sharma, S. K. Kumar, and S. K. Nayak, Phys. Rev. B 74, 035339 (2006).

${ }^{11}$ A. M. Smith, A. M. Mohs, and S. Nie, Nat. Nanotechnol. 4, 56 (2009).

${ }^{12}$ G. Hadjisavvas and P. C. Kelires, Physica E 38, 99 (2007).

${ }^{13}$ R. Guerra, I. Marri, R. Magri, L. Martin-Samos, O. Pulci, E. Degoli, and S. Ossicini, Phys. Rev. B 79, 155320 (2009).

${ }^{14}$ R. Tsu, H. Shen, and M. Dutta, Appl. Phys. Lett. 60, 112 (1992).

${ }^{15}$ T. Arguirov, T. Mchedlidze, M. Kittler, R. Rolver, B. Berghoff, M. Frost, and B. Spangenberg, Appl. Phys. Lett. 89, 053111 (2006).

${ }^{16}$ H. Coffin, C. Bonafos, S. Schamm, N. Cherkashin, G. B. Assayag, A. Claverie, M. Respaud, P. Dimitrakis, and P. Normand, J. Appl. Phys. 99, 044302 (2006).

${ }^{17}$ D. E. Yılmaz, C. Bulutay, and T. Çă̆ın, Phys. Rev. B 77, 155306 (2008)

${ }^{18}$ M. Ippolito, S. Meloni, and L. Colombo, Appl. Phys. Lett. 93, 153109 (2008).

${ }^{19}$ A. C. T. van Duin, A. Strachan, S. Stewman, Q. Zhang, X. Xu, and W. A. Goddard III, J. Phys. Chem. A 107, 3803 (2003).

${ }^{20}$ B. Joe, Adv. Eng. Software 13, 325 (1991).

${ }^{21}$ P. M. Gullett, M. F. Horstemeyer, M. I. Baskes, and H. Fang, Modell. Simul. Mater. Sci. Eng. 16, 015001 (2008).

${ }^{22}$ W. A. Curtin and R. E. Miller, Modell. Simul. Mater. Sci. Eng. 11, R33 (2003)

${ }^{23}$ C. Pryor, J. Kim, L. W. Wang, A. J. Williamson, and A. Zunger, J. Appl. Phys. 83, 2548 (1998).

${ }^{24}$ P. C. Chou and N. J. Pagano, Elasticity-Tensor, Dyadic and Engineering Approaches (Dover, Princeton, 1967).

${ }^{25}$ M. S. Majdoub, P. Sharma, and T. Cagin, Phys. Rev. B 77, 125424 (2008) 